\title{
Endosialin (CD248) is a marker of tumor-associated pericytes in high-grade glioma
}

\author{
Nicole Simonavicius ${ }^{1}$, David Robertson ${ }^{1}$, Dorine A Bax ${ }^{2}$, Chris Jones ${ }^{2}$, Ivo J Huijbers ${ }^{1}$ \\ and Clare $\mathrm{M}$ Isacke ${ }^{1}$ \\ ${ }^{1}$ Breakthrough Breast Cancer Research Centre, The Institute of Cancer Research, London, UK \\ and ${ }^{2}$ Department of Paediatric Oncology, The Institute of Cancer Research, Sutton, UK
}

\begin{abstract}
Gliomas are the most frequent primary tumors of the central nervous system in adults. The most prevalent and aggressive subclass of these is glioblastoma multiforme, which is characterized by massive neovascularization. Endosialin (CD248) has generated interest as a target for antiangiogenic therapy following reports that its expression is upregulated on angiogenic endothelial cells. We demonstrate here that endosialin is not expressed in normal human adult brain but is strongly upregulated in the angiogenic vasculature of all high-grade glioma specimens examined. However, by taking advantage of a technique which allows for multiple fluorescent labeling of formalin-fixed paraffin-embedded archival sections, we demonstrate unambiguously that endosialin is not expressed by the glioma endothelial cells but on closely associated perivascular cells. With increasing awareness that targeting pericytes is an attractive adjunct in antiangiogenic therapy, this finding has important implications for understanding the molecular mechanisms regulating angiogenesis in these highly vascularized tumors.
\end{abstract}

Modern Pathology (2008) 21, 308-315; doi:10.1038/modpathol.3801006; published online 11 January 2008

Keywords: endosialin; CD248; glioma; pericytes; perivascular cells; TEM1

Brain tumors account for approximately 20500 new cases and 13000 deaths each year in the United States. ${ }^{1}$ The most frequent primary brain tumors in adults are the gliomas, which represent a morphologically diverse set of malignancies diagnosed on the basis of their histology. These include diffuse astrocytomas (WHO grade II), anaplastic astrocytomas (WHO grade III) and glioblastomas (WHO grade IV). ${ }^{2}$ Glioblastoma multiforme is the most prevalent and aggressive type of glioma with a median survival of 12-15 months. ${ }^{3}$ Since standard therapy, especially in recurrent glioblastoma multiforme, has only limited impact on the survival of patients, ${ }^{4}$ a better understanding of the molecular alterations is needed to develop targeted treatment. Glioblastoma multiforme is considered an attractive target for antiangiogenic therapy, because one of its main characteristics is an extensive micro-

Correspondence: Dr IJ Huijbers, Breakthrough Breast Cancer Research Centre, The Institute of Cancer Research, 237 Fulham Road, London SW3 6JB, UK.

E-mail: ivo.huijbers@icr.ac.uk

Received 17 October 2007; revised 21 November 2007; accepted 26 November 2007; published online 11 January 2008 vasculature, in some cases leading to multilayered glomeruloid-type microvascular proliferations.

Endosialin (CD248) raised interest as a potential therapeutic antiangiogenic target after it was identified in a SAGE screen as the most highly upregulated transcript in endothelial cells isolated from colon carcinomas compared to endothelial cells isolated from neighboring normal tissue, hence its alternate name of tumor endothelial marker 1 or TEM $1 .^{5}$ Endosialin is a type 1 transmembrane glycoprotein originally identified as the target of the FB5 monoclonal antibody. ${ }^{6}$ The domain structure of endosialin, with a C-type lectin-like domain, a sushi domain, three epidermal growth factor repeats and a serine/threonine rich mucin-like stalk has placed it in a family of proteins together with CD93 (the complement receptor C1qRp) and CD141 (thrombomodulin). ${ }^{7}$ In cultured cells, expression of endosialin has only been reported on fibroblasts and some neuroblastoma cell lines. ${ }^{6-9}$ This pattern is reflected in normal adult tissue, where endosialin expression is predominantly restricted to stromal fibroblasts, and in agreement with the SAGE analysis of St Croix et $a l^{5}$ little or no endosialin is detected in the mature, normal vasculature. ${ }^{9-13}$ In contrast, there is a marked upregulation of 
endosialin expression in the angiogenic vasculature of a wide variety of different tumor types including those of the brain, colon, breast and in tumor xenografts. ${ }^{6,9,11,12,14,15}$ However, there is controversy between these reports as to whether this upregulation is due to expression by the endothelial cells and/or the perivascular cells. Two major difficulties have arisen in resolving this controversy. First, double labeling studies have traditionally required the staining of cryosections, in which the cell and tissue morphology are less well preserved. In addition, the availability of fresh tumor material required for cryosections is very limited in comparison to the large collections of formalin-fixed paraffin-embedded tissues. Second, the close proximity of stromal fibroblasts near the angiogenic vasculature has resulted in some debate as to whether expression of endosialin on cells adjacent to angiogenic endothelial cells represents expression on pericytes or closely associated fibroblasts.

To specifically address both these issues, we have examined the expression of endosialin in a series of high-grade gliomas. The benefits of using brain tissue are the low number of stromal fibroblasts and the presence of an extensive vasculature. In addition, we have employed an immunofluorescent labeling technique, which allows high-resolution multicolor imaging of formalin-fixed paraffinembedded sections. Together this has allowed us to demonstrate unambiguously that expression of endosialin in the angiogenic vasculature of highgrade gliomas is restricted to perivascular cells, and that endosialin is not detected on the endothelial cells.

\section{Materials and methods}

\section{Samples}

Brain tumor samples were obtained after approval by Local and Multicentre Ethical Review Committees from the Royal Marsden Hospital, Sutton, and St Georges Hospital Medical School, London, UK. In total, 10 high-grade gliomas were studied, comprising eight WHO grade IV glioblastoma multiforme, and two grade III anaplastic astrocytoma. The age range was from 31-72 years. Paraffinembedded normal adult brain from individuals with no history of neurological disease was provided by Dr Peter Clark, Imperial College London from the UK Multiple Sclerosis Tissue Bank, Imperial College London, and was processed using standard histological techniques. ${ }^{16}$

\section{Antibodies and Cells}

The generation of the human anti-endosialin monoclonal antibodies (B1/35 and B1/22) has been described previously. ${ }^{9}$ The following antibodies were obtained commercially: anti- $\alpha$-tubulin (clone:
B-5-1-2, Sigma-Aldrich, St Louis, MO, USA), anti-human- $\alpha$-smooth muscle actin (clone: 1A4, Sigma-Aldrich), anti-human-CD34 monoclonal antibody (clone: QBEND 10, Sky Bio, Bedfordshire, UK), fluorescein isothiocyanate-conjugated anti-human CD34 monoclonal antibody (clone: QBEND 10, Abcam, Cambridge, UK), Horseradish peroxidase (HRP)-anti-mouse Ig (Jackson Immunoresearch, West Grove, PA, USA), Alexa-conjugated second layer antibodies (Molecular Probes, Eugene, OR, USA). Diploid human AG1523 fibroblasts and human MCF7 epithelial breast carcinoma cells were cultured in Dulbecco's modified Eagle's medium supplemented with $10 \%$ fetal bovine serum, $2 \mathrm{mM}$ L-glutamine and penicillin/streptomycin. Human MCF10A mammary epithelial cells were grown in Dulbecco's modified Eagle's medium:F12, supplemented with $5 \%$ horse serum, $10 \mu \mathrm{M}$ HEPES, $10 \mu \mathrm{g} /$ $\mathrm{ml}$ insulin, $20 \mathrm{ng} / \mathrm{ml} \mathrm{EGF,} 0.5 \mu \mathrm{g} / \mathrm{ml}$ hydrocortisone, $100 \mathrm{ng} / \mathrm{ml}$ cholera toxin. Cells were cultured at $37^{\circ} \mathrm{C}$ in a tissue culture incubator with humidified air, supplemented with $\mathrm{CO}_{2}$ to $10 \%$.

\section{Western Blot Analysis}

Cells were lysed in NuPage non-reducing sample buffer and run on $10 \%$ SDS-PAGE gels. Gels were transferred onto nitrocellulose membrane, blocked with $5 \%$ low-fat milk and incubated with the primary antibodies: B1/35, B1/22 $(0.2 \mu \mathrm{g} / \mathrm{ml})$ and $\alpha$-tubulin (1:30000 dilution), followed by horseradish peroxidase-conjugated anti-mouse IgG $(0.2 \mu \mathrm{g} / \mathrm{ml})$. Immunodetection was carried out using the ECL Kit (Amersham, Buckhinghamshire, UK) followed by exposure to X-ray film (Kodak, Rochester, NY, USA) for $20 \mathrm{~min}$.

\section{Immunohistochemistry}

Formalin-fixed paraffin-embedded sections (3 or $4 \mu \mathrm{m})$ were dewaxed in xylene $(2 \times 5 \mathrm{~min})$ and rehydrated through a series of graded alcohols to water. Slides were then pressure cooked for $2 \mathrm{~min}$ in $0.01 \mathrm{M} \mathrm{pH} 6.0$ citrate target retrieval buffer, washed 5 min under running water and stained with the anti-endosialin monoclonal antibodies (B1/35, B1/ $22,2 \mu \mathrm{g} / \mathrm{ml}$ ) or with anti-CD34 monoclonal antibodies at a 1:50 dilution for $1 \mathrm{~h}$ at room temperature. Detection was achieved with the Vectastain avidinbiotin complex (ABC) system according to the manufacturer's protocol (Vector Laboratories, Burlingame, CA, USA). Finally slides were dehydrated through a series of alcohols, cleared in xylene and mounted in 1,3-diethyl-8-phenylxanthine. Sections of human placenta served as positive control for stainings. Images were captured on a Leica DMRA2 microscope fitted with a Leica DFC320 camera and processed with Adobe Photoshop 7.0. All images were taken at the same camera settings. 


\section{Confocal Microscopy}

Staining of cells on glass coverslips was performed as described previously. ${ }^{13}$ For confocal microscopy of formalin-fixed paraffin-embedded material, sections were dewaxed and rehydrated and then antigen retrieval was performed at $95^{\circ} \mathrm{C}$ in DAKO Cytomation target retrieval solution (DAKO, Denmark) for $30 \mathrm{~min}$ followed by $20 \mathrm{~min}$ at room temperature and $5 \mathrm{~min}$ under running water. Slides were removed from water, sections marked using a slide marker pen and incubated with primary antibodies for $1 \mathrm{~h}$ at room temperature. After $3 \times 5$ min wash with PBS the appropriate secondary antibodies were incubated for $1 \mathrm{~h}$ at room temperature. For double labeling of endosialin and CD34, staining was performed in the following order: B1/35 $(2 \mu \mathrm{g} / \mathrm{ml})$, Alexa555 anti-mouse Ig $(2 \mu \mathrm{g} / \mathrm{ml})$, FITC-conjugated CD34 monoclonal antibody $(2.5 \mu \mathrm{g} / \mathrm{ml})$. For double labeling of endosialin and $\alpha$-smooth muscle actin, slides were incubated with B1/35 $(2 \mu \mathrm{g} / \mathrm{ml})$ and anti- $\alpha$-smooth muscle actin $(0.88 \mu \mathrm{g} / \mathrm{ml})$ followed by the $2 \mu \mathrm{g} / \mathrm{ml}$ Alexa555conjugated anti-mouse- $\operatorname{IgG}_{1}$ (to detect monoclonal antibody B1/35) and $2 \mu \mathrm{g} / \mathrm{ml}$ Alexa488-anti-mouse$\mathrm{IgG}_{2 \mathrm{~A}}$ (to detect the anti- $\alpha$-smooth muscle actin monoclonal antibody). Control experiments showed that there was no non-specific binding or crossreactivity of the secondary antibodies. Nuclei were counterstained with $14 \mathrm{nM}$ DAPI (Molecular Probes), slides were rinsed in PBS, mounted with Vectashield (Vector Laboratories) and the coverslips sealed with nail varnish. Fluorescent images were collected sequentially in three channels on a Leica Microsystems (Leica, Wetzlar, Germany) TCS-SP2 confocal microscope. All images were taken at the same settings. Images were exported from the Leica software and processed with Adobe Photoshop 7.0. A full method describing the immunofluorescent staining of formalin-fixed paraffin-embedded sections has been submitted for publication (Robertson D, Savage K, Reis-Filho JS and Isacke CM).

\section{Results}

\section{Characterization of the Human Endosialin Monoclonal Antibodies}

We have previously described four monoclonal antibodies which recognize human endosialin in immunoprecipitation and immunofluorescence experiments. ${ }^{9}$ Here, we have further characterized two of these anti-endosialin monoclonal antibodies, B1/35 and B1/22 by immunoblotting and immunofluorescent staining. Western blots on lysates of AG1523 human fibroblasts, which endogenously express endosialin, demonstrated that both monoclonal antibodies recognize the mature $175 \mathrm{kDa}$ protein and a lower $80 \mathrm{kDa}$ molecular weight band (Figure 1a). This banding pattern has been reported previously for both human and mouse endosialin

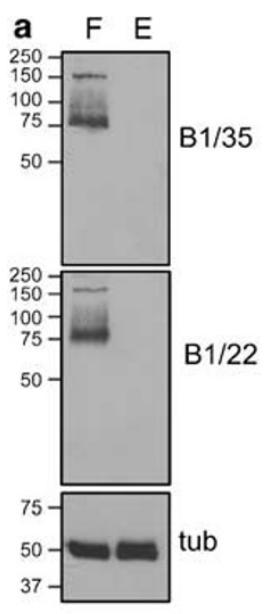

b

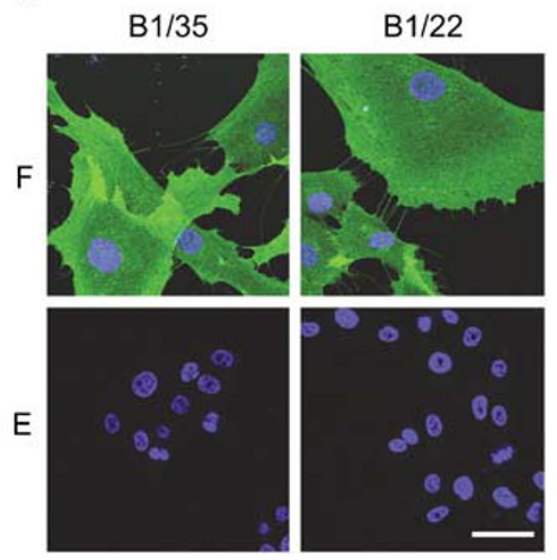

c

$\mathrm{B} 1 / 35$
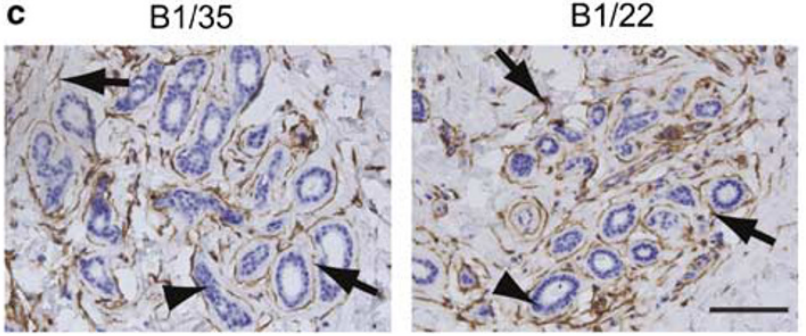

Figure 1 Characterization of the human endosialin monoclonal antibodies. (a) Lysates of AG1523 human fibroblasts (F) and MCF7 human epithelial cells (E) were subject to immunoblotting using either monoclonal antibody B1/35, monoclonal antibody $\mathrm{B} 1 / 22$ or an anti- $\alpha$-tubulin (tub) monoclonal antibody as a loading control. (b) AG1523 fibroblasts (F) and MCF10A epithelial cells (E) were fixed in $4 \%$ paraformaldehyde, permeabilized with $0.5 \%$ Triton X-100 and stained with the monoclonal antibodies B1/35 or B1/22 followed by Alexa488-anti-mouse IgG (green) and counterstained with TO-PRO-3 (blue). Scale bar, $50 \mu \mathrm{m}$. (c) Formalin-fixed paraffin-embedded sections of normal breast were stained with the monoclonal antibody B1/35 or B1/22 and counterstained with haematoxylin. Arrows indicate endosialinexpressing fibroblasts. Arrowheads indicate endosialin-negative epithelial cells. Scale bar, $150 \mu \mathrm{m}$.

detected with other monoclonal antibodies and polyclonal antibodies. ${ }^{7,8,12,13}$ The $80 \mathrm{kDa}$ band most likely represents a population of intracellular immaturely glycosylated endosialin as both these monoclonal antibodies have previously been shown to only recognize the $175 \mathrm{kDa}$ endosialin following immunoprecipitation of cell surface labeled human fibroblasts. ${ }^{9}$ Neither monoclonal antibody demonstrated any reactivity with lysates from endosialinnegative MCF7 cells (Figure 1a). In immunofluorescent staining of cells in culture, B1/35 and B1/22 showed an identical granular distribution of endosialin on the plasma membrane of AG1523 fibroblasts but showed no staining of MCF10A epithelial cells (Figure 1b). Finally to assess the ability of these monoclonal antibodies to recognize endosialin in formalin-fixed paraffin-embedded material, sections of normal human breast were stained. As shown in Figure 1c, both antibodies were able to detect endosialin on the interlobular and intralobular fibroblasts but not on either the 
a
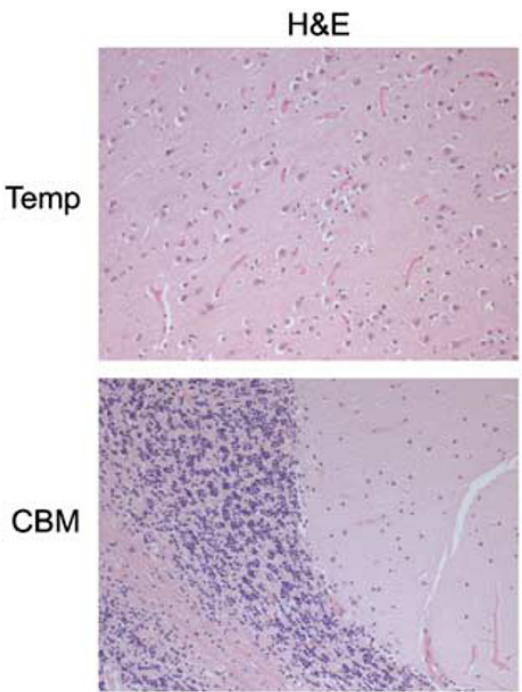

b

3314 (III)
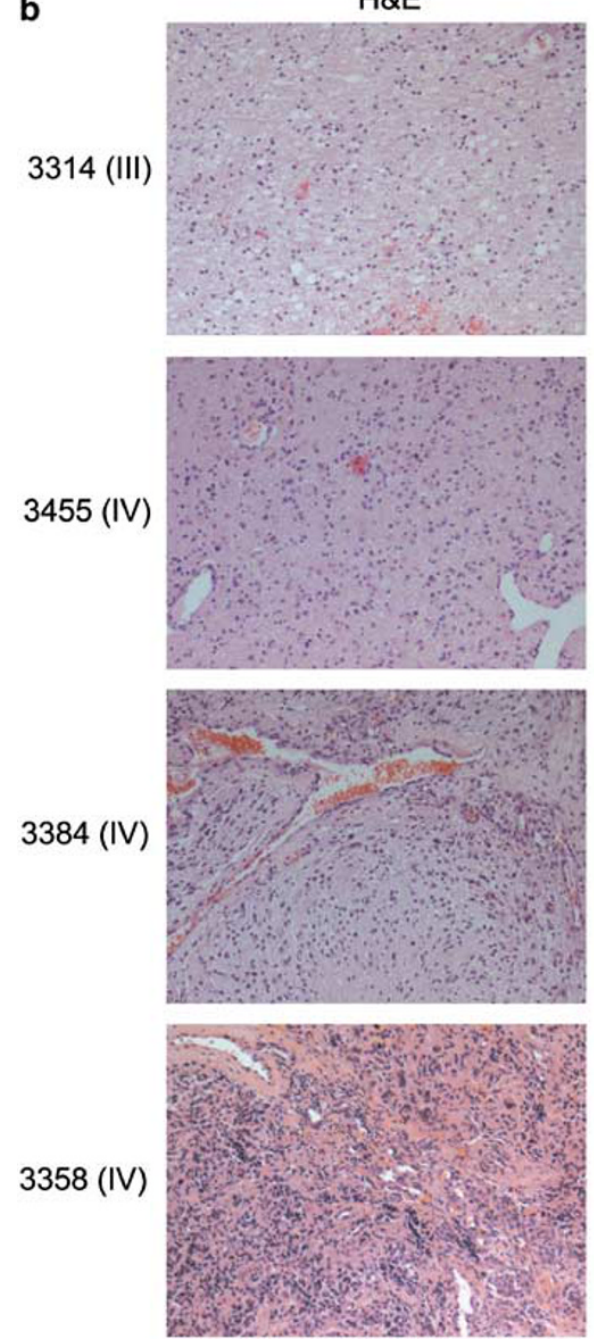

B1/35
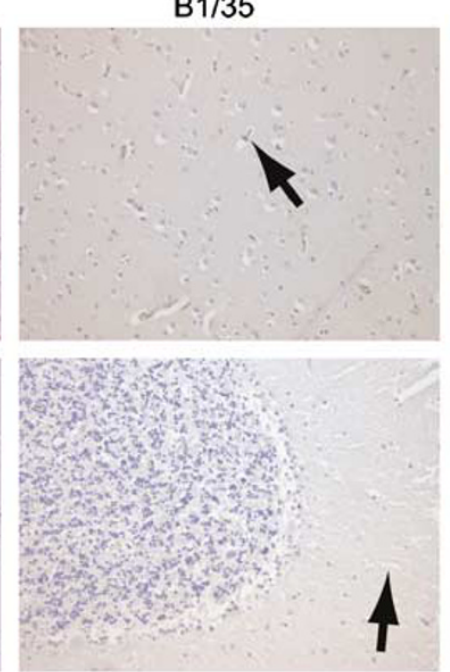

B1/35
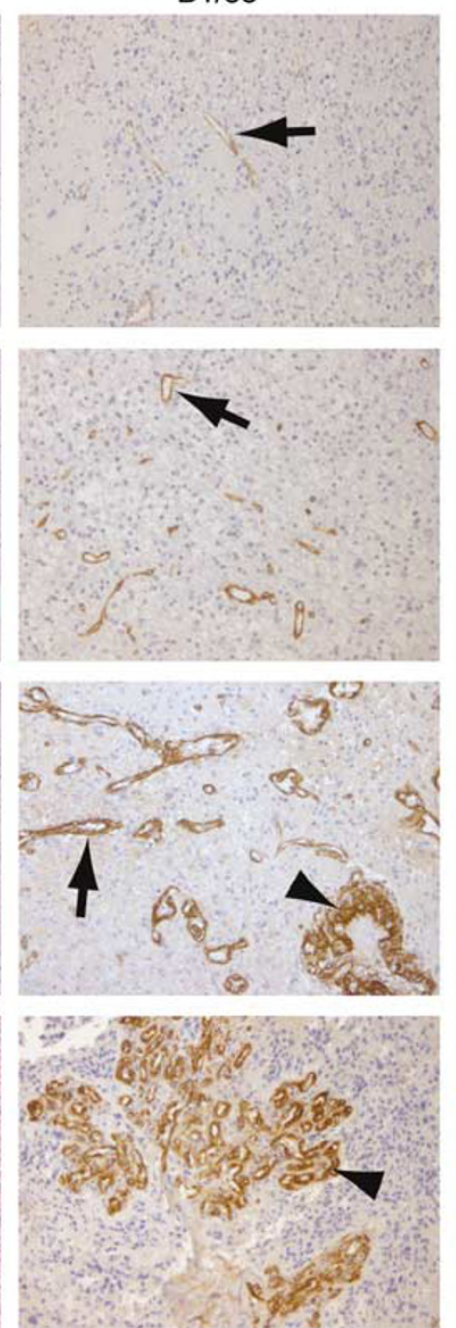

\section{CD34}
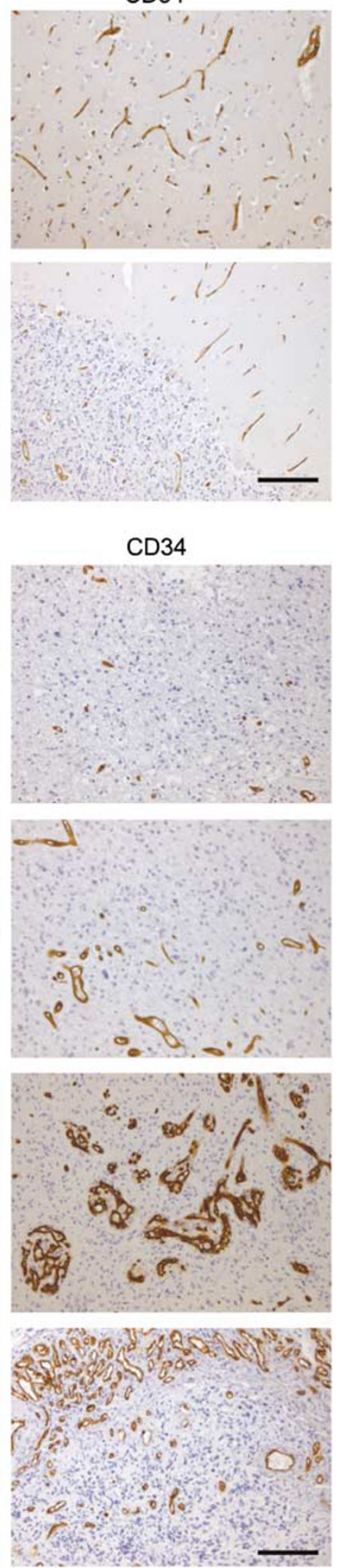

Figure 2 Histopathological assessment of endosialin expression in normal adult human brain and high-grade gliomas. Formalin-fixed paraffin-embedded sections were stained with haematoxylin and eosin (H\&E), the anti-endosialin monoclonal antibody B1/35 or with anti-CD34 monoclonal antibody. (a) Temporal lobe (Temp) and cerebellum (CBM) of normal adult human brain. Arrows indicate endosialin-negative vessels. Scale bar, $150 \mu \mathrm{m}$. (b) Representative images from 1 grade III tumor (3314) and 3 grade IV tumors (3455, 3384, and 3358). Arrows indicate endosialin-positive vessels. Arrowheads indicate strongly stained glomeruloid microvascular proliferations. Results from all 10 high-grade glioblastomas are summarized in Table 1. Scale bar, $150 \mu \mathrm{m}$. 
myoepithelial or luminal epithelial cells. This expression is identical to that previously reported following staining of endosialin on normal breast cryosections. ${ }^{9}$ As no differences in reactivity in any of these assays were observed between the independently isolated $\mathrm{B} 1 / 35$ and $\mathrm{B} 1 / 22$ anti-endosialin monoclonal antibodies, only B1/35 was used for further experiments.

\section{Endosialin Expression is Upregulated on the Vasculature of High-Grade Gliomas}

To assess expression of endosialin in normal adult human brain and high-grade gliomas, formalin-fixed paraffin-embedded sections were stained with the anti-endosialin monoclonal antibody B1/35 and parallel sections were stained for the endothelial marker CD34. In normal brain, the vasculature was detected with the anti-CD34 monoclonal antibody but no staining was observed on the vasculature or on any other cell types with B1/35 (Figure 2a). In contrast, endosialin expression was readily detected in the vasculature of 10 different highgrade gliomas, with stronger staining observed in the 8 grade IV gliomas compared to the 2 grade III anaplastic astrocytomas (Table 1, Figure 2b). In the higher grade tumors, endosialin expression was detected on the small vessels and the glomeruloid microvascular proliferations.

\section{Endosialin is Expressed by Pericytes in High-Grade Gliomas but not by Endothelial Cells}

To better assess the cell type distribution of endosialin in the tumor vasculature, formalin-fixed paraffin-embedded sections were double labeled for endosialin and markers of either endothelial or perivascular cells and images were collected by confocal microscopy. Using this high resolution imaging method, it was clearly demonstrated that there was no overlap in staining of endosialin and the endothelial marker CD34 (Figure 3a). To verify the location of endosialin on cells adjacent to the endothelium, sections were costained with the pericyte marker, $\alpha$-smooth muscle actin. As illustrated in Figure $3 \mathrm{~b}$, the majority of endosialinpositive cells also stained positive for $\alpha$-smooth muscle actin and in addition, a small number of dispersed endosialin-positive, $\alpha$-smooth muscle actin-negative stromal fibroblasts were also identified.

\section{Discussion}

To date there has been a controversy in the literature as to the cell type expression of endosialin in the tumor vasculature. Endosialin was first identified as the epitope for monoclonal antibody $\mathrm{FB}^{6}$ and staining with this antibody revealed an upregulation of endosialin expression in the vasculature of a
Table 1 Summary of endosialin immunohistochemical staining in normal brain and in 10 high grade gliomas

\begin{tabular}{lcc}
\hline Sample number & WHO grade & Endosialin (B1/35) \\
\hline Normal brain (CBM) & NA & 0 \\
Normal brain (Temp) & NA & 0 \\
3314 & III & $1+$ \\
3365 & III & $2+$ \\
3312 & IV & $\mathrm{G} 3+$ \\
3317 & IV & $\mathrm{G} 3+$ \\
3358 & IV & $\mathrm{G} 3+$ \\
3384 & IV & $\mathrm{G} 3+$ \\
3391 & IV & $\mathrm{G} 3+$ \\
3401 & IV & $3+$ \\
3431 & IV & $2+$ \\
3455 & IV & $2+$ \\
\end{tabular}

CBM, cerebellum; Temp, temporal lobe.

Staining was scored as negative (0), weak (1+), moderate $(2+)$ and strong $(3+)$. G3+ indicates tumors with strong endosialin staining in the glomeruloid microvascular proliferations. Example images are shown in Figures 2 and 3.

variety of human tumors. Despite reporting that this represented expression on the endothelial cells, the cell type distribution was not validated by double labeling with endothelial markers. Similarly, in situ hybridization analysis has demonstrated an upregulation of endosialin mRNAs in the tumor vasculature but again this method cannot distinguish between an endothelial and perivascular localization of the signal. ${ }^{5,14,17}$ As endosialin is also expressed in the developing vasculature, different laboratories have performed double labeling of cryosections both from tumors and developing tissues. However, disparate results have been obtained in that it has been reported that endosialin is only expressed on the endothelial cells, ${ }^{12}$ only on the pericytes ${ }^{9,13,18}$ and on both. ${ }^{15}$ The main problem in such analysis is that in tissue cryosections it can be difficult to distinguish between an overlapping staining pattern and staining of individual cells which are intimately associated, as is the case for endothelial cells and pericytes.

To address this controversy, we first characterized further two anti-endosialin monoclonal antibodies, $\mathrm{B} 1 / 35$ and B1/22. Both antibodies showed an identical cell surface staining and western blotting pattern on cultured human fibroblasts and importantly both antibodies were able to detect endosialin in formalin-fixed paraffin-embedded sections. Monoclonal antibody B1/35 was then used to characterize the expression of endosialin in normal brain and in high-grade gliomas. In agreement with previous studies in the mouse brain and human brain tumor samples, ${ }^{12,13,15}$ no expression of endosialin was detected in the normal adult human brain but expression was upregulated in the vasculature of all high-grade gliomas examined. Highest levels of expression were found in the grade IV gliomas and in particular on the glomeruloid microvascular proliferations. These are tufted collections of newly formed vascular channels containing hyperplastic 
a
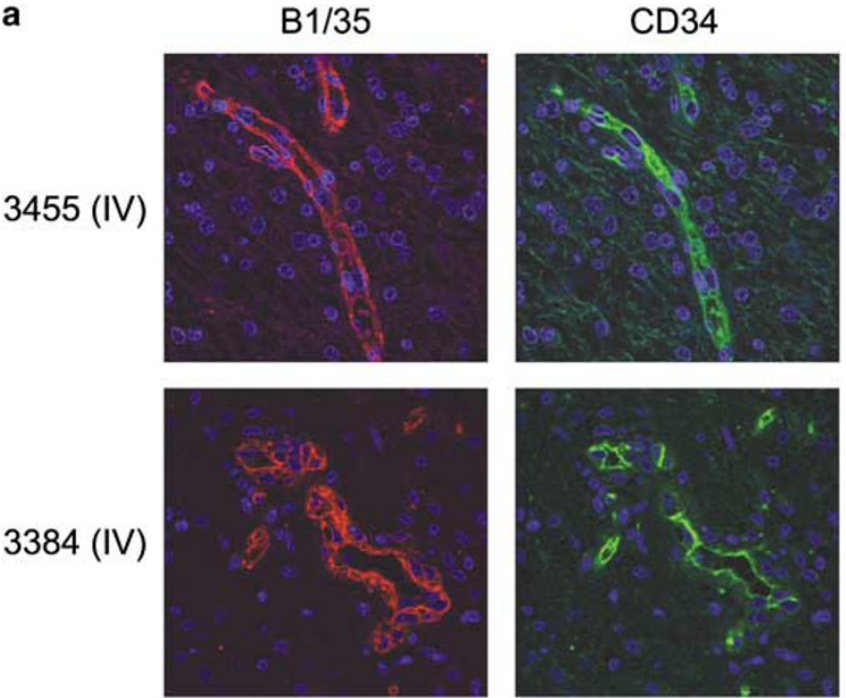

3384 (IV)
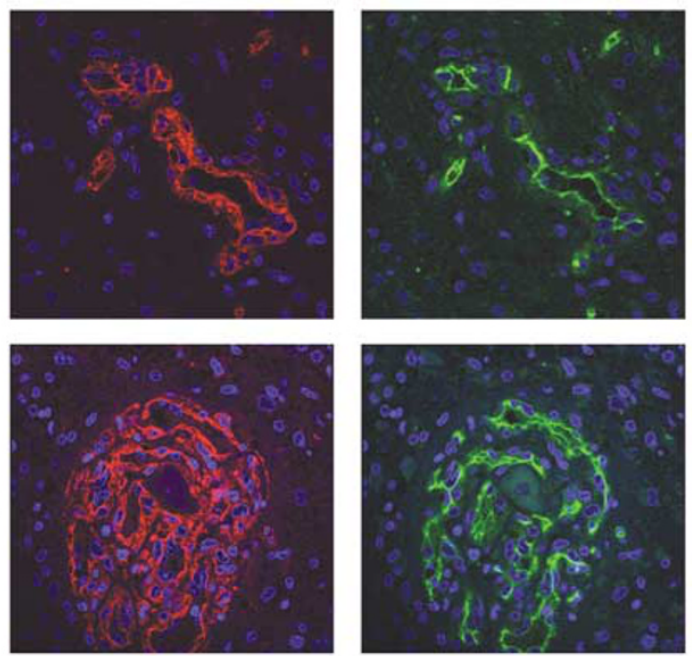

b

B1/35

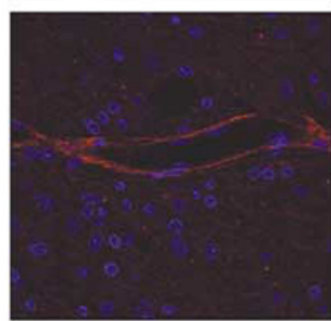

3455 (IV)

3384 (IV)

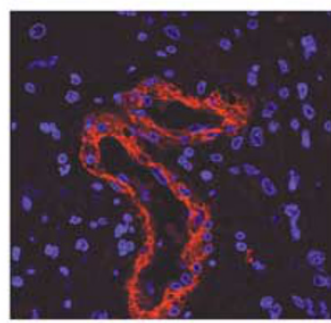

3321 (IV)

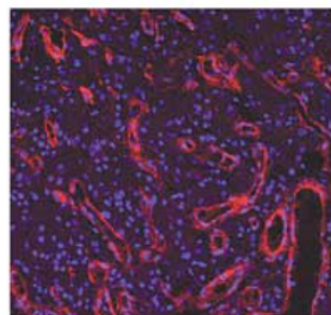

$\alpha \mathrm{SMA}$
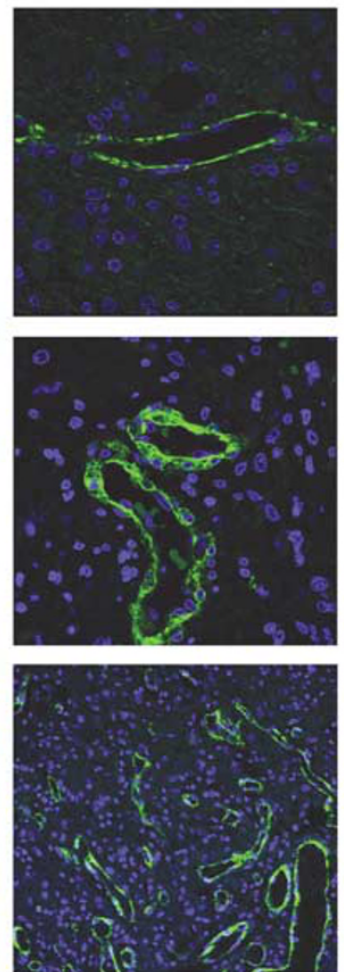
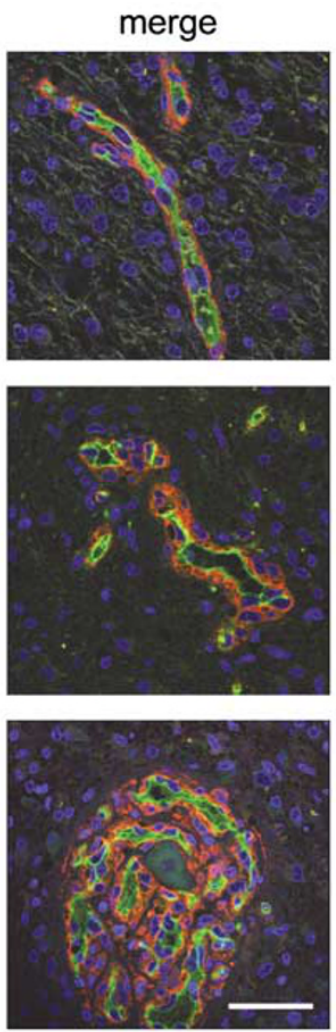

merge
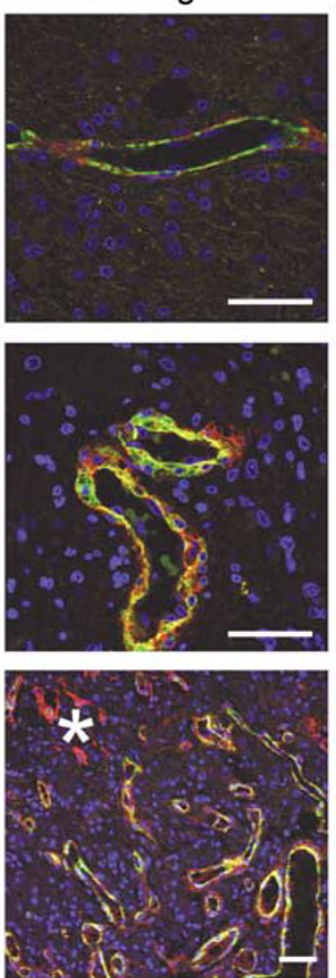

merge $\times 2.5$
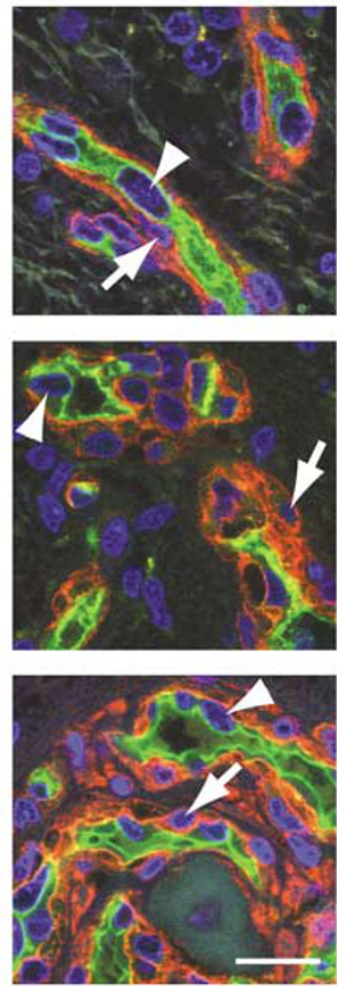

merge $\times 2.5$
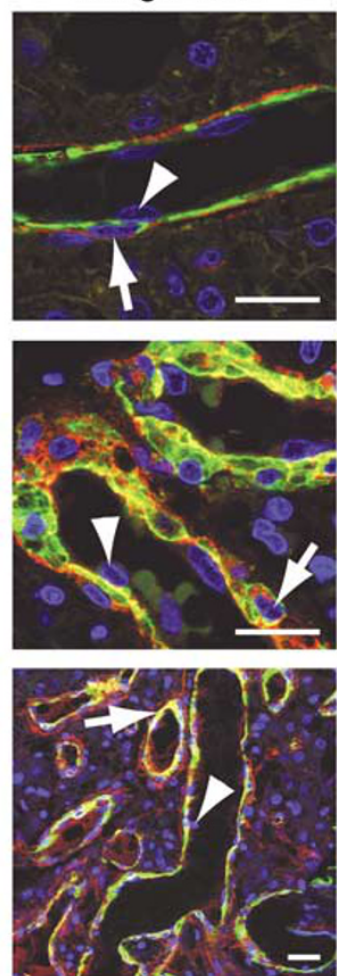

Figure 3 Distribution of endosialin on tumor-associated vessels in high-grade gliomas. Formalin-fixed paraffin-embedded sections of grade IV gliomas were costained with (a) monoclonal antibody B1/35 (red) and anti-CD34 (green) or (b) monoclonal antibody B1/35 (red) and anti- $\alpha$-smooth muscle actin (green). Nuclei were counterstained with DAPI (blue). Arrowheads indicate endothelial cells. Arrows indicate perivascular cells. *Indicates endosialin-positive, $\alpha$-smooth muscle actin-negative fibroblasts. Scale bar, $50 \mu \mathrm{m}$ in main panels and $20 \mu \mathrm{m}$ in merge $\times 2.5$ panels. 
endothelial cells surrounded by numerous pericytes and a basal lamina. ${ }^{19}$ Next, we combined immunofluorescent double labeling of formalin-fixed paraffin-embedded sections with high resolution confocal imaging of thin $(\sim 0.5 \mu \mathrm{m})$ optical sections. Costaining of these sections with the anti-endosialin monoclonal antibody B1/35 and with an antibody against the endothelium-specific marker CD34 revealed a clear separation of the two antibodies, with the endosialin-positive cells lying adjacent to the endothelial cells. This separation of staining was detected in both the smaller vessels and the larger complex glomeruloid microvascular proliferations. Double labeling of sections with antibodies against endosialin and the frequently used pericyte marker $\alpha$-smooth muscle actin, ${ }^{20-22}$ confirmed that endosialin is expressed by $\alpha$-smooth muscle actin-positive pericytes.

In conclusion, endosialin is not expressed in the vasculature of normal adult brain but strongly upregulated in tumor vessels in high-grade gliomas. By employing confocal microscopy of immunolabeled formalin-fixed paraffin-embedded sections, it was unambiguously shown that the expression of endosialin is restricted to perivascular cells and some scattered fibroblasts, validating endosialin as a robust pericyte marker. The significance of these findings is twofold. First, mice with a homozygous deletion in endosialin (Cd248) show impaired growth, invasion and vascularization of implanted abdominal tumors, ${ }^{17}$ suggesting that endosialin expression on pericytes has an important functional role in the tumor vasculature. Second, there is increasing evidence that antiangiogenic therapies targeting both endothelial cells and pericytes are more effective than single-agent therapies. ${ }^{23}$ Endosialin, unlike other pericyte makers, is not expressed in the normal vasculature, but strongly upregulated in the tumor vasculature. As a consequence targeting endosialin or pathways associated with this receptor offer an attractive therapeutic opportunity. This may be particularly relevant to glioblastoma multiforme, which is characterized by extensive microvascular proliferation, but for which there is currently little targeted treatments.

\section{Acknowledgements}

We thank Dr Darren Hargrave (Royal Marsden Hospital) and Dr Peter Wilkins (St Georges Hospital Medical School) for providing the high-grade glioma samples and clinicopathological data, Peter Clark (Imperial College, London) for providing the normal brain samples, and Kay Savage and Suzanne Parry (Breakthrough Histopathology Laboratory, Institute of Cancer Research) for immunohistochemical staining of the sections. This work was funded by a grant from Breakthrough Breast Cancer (CMI) and Cancer Research UK (CJ).

\section{Disclosure/duality of interest}

There are no disclosures or duality of interests to declare.

\section{References}

1 Jemal A, Siegel R, Ward E, et al. Cancer statistics, 2007. CA Cancer J Clin 2007;57:43-66.

2 Louis DN, Ohgaki H, Wiestler OD, et al. The 2007 WHO classification of tumours of the central nervous system. Acta Neuropathol (Berl) 2007;114:97-109.

3 Stupp R, Mason WP, van den Bent MJ, et al. Radiotherapy plus concomitant and adjuvant temozolomide for glioblastoma. N Engl J Med 2005;352:987-996.

4 Simpson L, Galanis E. Recurrent glioblastoma multiforme: advances in treatment and promising drug candidates. Expert Rev Anticancer Ther 2006;6: 1593-1607.

5 St Croix B, Rago C, Velculescu V, et al. Genes expressed in human tumor endothelium. Science 2000;289: 1197-1202.

6 Rettig WJ, Garin-Chesa P, Healey JH, et al. Identification of endosialin, a cell surface glycoprotein of vascular endothelial cells in human cancer. Proc Natl Acad Sci USA 1992;89:10832-10836.

7 Christian S, Ahorn H, Koehler A, et al. Molecular cloning and characterization of endosialin, a C-type lectin-like cell surface receptor of tumor endothelium. J Biol Chem 2001;276:7408-7414.

8 Opavsky R, Haviernik P, Jurkovicova D, et al. Molecular characterization of the mouse Tem1/endosialin gene regulated by cell density in vitro and expressed in normal tissues in vivo. J Biol Chem 2001;276: 38795-38807.

9 MacFadyen JR, Haworth O, Roberston D, et al. Endosialin (TEM1, CD248) is a marker of stromal fibroblasts and is not selectively expressed on tumour endothelium. FEBS Lett 2005;579:2569-2575.

10 Dolznig H, Schweifer N, Puri C, et al. Characterization of cancer stroma markers: in silico analysis of an mRNA expression database for fibroblast activation protein and endosialin. Cancer Immun 2005;5:10.

11 Huber MA, Kraut N, Schweifer N, et al. Expression of stromal cell markers in distinct compartments of human skin cancers. J Cutan Pathol 2006;33:145-155.

12 Rupp C, Dolznig H, Puri C, et al. Mouse endosialin, a C-type lectin-like cell surface receptor: expression during embryonic development and induction in experimental cancer neoangiogenesis. Cancer Immun 2006;6:10.

13 MacFadyen J, Savage K, Wienke D, et al. Endosialin is expressed on stromal fibroblasts and CNS pericytes in mouse embryos and is downregulated during development. Gene Expr Patterns 2007;7:363-369.

14 Carson-Walter EB, Watkins DN, Nanda A, et al. Cell surface tumor endothelial markers are conserved in mice and humans. Cancer Res 2001;61:6649-6655.

15 Brady J, Neal J, Sadakar N, et al. Human endosialin (tumor endothelial marker 1) is abundantly expressed in highly malignant and invasive brain tumors. J Neuropathol Exp Neurol 2004;63:1274-1283.

16 Williams MJ, Lowrie MB, Bennett JP, et al. Cadherin-10 is a novel blood-brain barrier adhesion molecule in human and mouse. Brain Res 2005;1058:62-72. 
17 Nanda A, Karim B, Peng Z, et al. Tumor endothelial marker 1 (Tem1) functions in the growth and progression of abdominal tumors. Proc Natl Acad Sci USA 2006;103:3351-3356.

18 Virgintino D, Girolamo F, Errede M, et al. An intimate interplay between precocious, migrating pericytes and endothelial cells governs human fetal brain angiogenesis. Angiogenesis 2007;10:35-45.

19 Kargiotis O, Rao JS, Kyritsis AP. Mechanisms of angiogenesis in gliomas. J Neurooncol 2006;78: 281-293.

20 Haddad SF, Moore SA, Schelper RL, et al. Vascular smooth muscle hyperplasia underlies the formation of glomeruloid vascular structures of glioblastoma multiforme. J Neuropathol Exp Neurol 1992;51: 488-492.
21 Wesseling P, Vandersteenhoven JJ, Downey BT, et al. Cellular components of microvascular proliferation in human glial and metastatic brain neoplasms. A light microscopic and immunohistochemical study of formalin-fixed, routinely processed material. Acta Neuropathol (Berl) 1993;85:508-514.

22 Wesseling $\mathrm{P}$, Schlingemann RO, Rietveld FJ, et al. Early and extensive contribution of pericytes/vascular smooth muscle cells to microvascular proliferation in glioblastoma multiforme: an immuno-light and immuno-electron microscopic study. J Neuropathol Exp Neurol 1995;54:304-310.

23 Bergers G, Song S, Meyer-Morse N, et al. Benefits of targeting both pericytes and endothelial cells in the tumor vasculature with kinase inhibitors. J Clin Invest 2003;111:1287-1295. 